\title{
Controlled atomic solubility in Mn-rich composite material to achieve superior electrochemical performance for $\mathrm{Li}$-ion batteries
}

\author{
Junghwa Lee1, Qinghua Zhang ${ }^{2}$, Jieun Kim¹, Nicolas Dupre ${ }^{3}$, Maxim Avdeev ${ }^{4,5}$, Mihee \\ Jeong $^{6}$, Won-Sub Yoon ${ }^{6}$, Lin Gu ${ }^{2,7,8}$, Byoungwoo Kang ${ }^{*}$ \\ ${ }^{1}$ Department of Materials Science and Engineering, Pohang University of Science and \\ Technology (POSTECH), Pohang 37673, Republic of Korea \\ ${ }^{2}$ Beijing National Laboratory for Condensed Matter Physics, Institute of Physics, Chinese \\ Academy of Sciences, Beijing 100190, China \\ ${ }^{3}$ Université de Nantes, Institut des Materiaux Jean Rouxel (IMN), CNRS UMR 6502, 2 rue de \\ la Houssiniere, BP 32229, 44322 Nantes Cedex 3, France \\ ${ }^{4}$ Australian Nuclear Science and Technology Organisation, Locked Bag 2001, Kirrawee DC, \\ NSW 2232, Australia \\ ${ }^{5}$ School of Chemistry, The University of Sydney, Sydney, NSW 2006, Australia \\ ${ }^{6}$ Department of Energy Science, Sungkyunkwan University, Suwon 16419, Republic of \\ Korea \\ ${ }^{7}$ Collaborative Innovation Center of Quantum Matter, Beijing 100190, China. \\ ${ }^{8}$ School of Physical Sciences, University of Chinese Academy of Sciences, Beijing 100190, \\ China. \\ * Corresponding author: bwkang@postech.ac.kr
}

The impending quest for high energy density and high power density electrode materials for lithium-ion batteries has been intensified to meet strongly growing demand for powering electric vehicles. Conventional layered oxides such as Co-rich $\mathrm{LiCoO}_{2}$ and Ni-rich $\mathrm{Li}\left(\mathrm{Ni}_{\mathrm{x}} \mathrm{Mn}_{\mathrm{y}} \mathrm{Co}_{\mathrm{z}}\right)_{2}$ that rely on only transition metal redox reaction have been faced with growing constraints on the resources and the availability due to soaring price on cobalt. Therefore, Mn-rich electrode materials excluding cobalt would be desirable with respect to available resources and low cost. Here, we report on the strategy of achieving both high energy density and high power density in Mn-rich electrode materials by controlling of the atoms solubility between phases in a composite. The resulting Mn-rich material that is composed of defective spinel phase and partially cation-disordered layered phase can achieve the highest 
energy density, $\sim 1100 \mathrm{~W} \cdot \mathrm{h} / \mathrm{kg}$ with superior power capability up to $10 \mathrm{C}$ rate $(3 \mathrm{~A} / \mathrm{g}$ ) among other reported Mn-rich materials. This approach provides new opportunities to design Mn-rich electrode materials that can achieve high energy density and high power density for Li-ion batteries.

Li-ion battery (LIB) has been an essential energy storage technology for powering advanced portable electronics. Now, the use of LIB has been extended to electric vehicles and grid-scale energy storage systems (ESS). Especially, large-scale energy storage systems have become a key player for transformational changes in our society by deploying smart grids that can make the consumption and distribution of energy efficient and by increasing the use of renewable resources such as solar and wind energy. For developing efficient large-scale energy storage systems, the cost-effective electrode materials with high energy density for Li-ion batteries are necessary. ${ }^{1}$ However, most of commercialized Li-ion cathode materials are based on only a few transition metals such as $\mathrm{Co}$ and $\mathrm{Ni}$, which is electroactive in layered electrode materials such as $\mathrm{LiCoO}_{2}$ and Ni-rich $\mathrm{Li}(\mathrm{Ni}, \mathrm{Mn}, \mathrm{Co}) \mathrm{O}_{2}$, causing constraints on their resources and availability. To decrease this strong dependence of electrode materials on Co or $\mathrm{Ni}$, high capacity electrode materials that are based on cheap and abundant raw materials are urgently needed. One of the most promising approach for this is the replacement of Co or Ni with cheap and abundant $\mathrm{Mn}$ such as Mn-rich electrode materials that can contain more than $80 \% \mathrm{Mn}$ in the total transition metal ions. ${ }^{2,3}$

To achieve high energy density in Mn-rich electrode materials, an increase in the use of the $\mathrm{Mn}$ redox reaction has been proposed. For example, $\mathrm{Li}_{4} \mathrm{Mn}_{2} \mathrm{O}_{5}$ with disordered-rocksalt structure can achieve the highest discharge capacity of $\sim 355 \mathrm{~mA} \cdot \mathrm{h} / \mathrm{g}$ with relatively low average potential $<3.0 \mathrm{~V}$ by using the $\mathrm{Mn}^{3+} / \mathrm{Mn}^{5+}$ double redox reaction that can be enabled 
by nanosized particles prepared by mechanochemical process. ${ }^{3}$ Also, recent reported disordered-rocksalt Mn-rich electrode materials such as $\mathrm{Li}_{2} \mathrm{Mn}_{2 / 3} \mathrm{Nb}_{1 / 3} \mathrm{O}_{2} \mathrm{~F}$ and $\mathrm{Li}_{2} \mathrm{Mn}_{1 / 2} \mathrm{Ti}_{1 / 2} \mathrm{O}_{2} \mathrm{~F}$ can achieve higher energy density than conventional layered materials by using the $\mathrm{Mn}^{2+} / \mathrm{Mn}^{4+}$ double redox that can be enabled by a fluorination process via the mechanochemical process and high-valency dopants such as $\mathrm{Nb}^{5+}$ and $\mathrm{Ti}^{4+} \cdot{ }^{2}$ However, these reported Mn-rich electrode materials suffer from sluggish Li diffusion resulting in poor power density because of the disordered-rocksalt structure that can have full random distribution of Li and transition metals (TMs) causing zig-zag-type short-range Li diffusion channel. The other approaches for improving electrochemical performance in Mn-rich electrode materials are the increase in an use of oxygen redox reactions by using the composite of spinel-layered phases; $x \mathrm{Li}\left[\mathrm{Mn}_{1.5} \mathrm{Ni}_{0.5}\right] \mathrm{O}_{4} \cdot(1-x)\left\{\mathrm{Li}_{2} \mathrm{MnO}_{3} \cdot \mathrm{Li}\left(\mathrm{Mn}_{0.5} \mathrm{Ni}_{0.5}\right) \mathrm{O}_{2}\right.$ or $\left.\mathrm{Li}_{2} \mathrm{MnO}_{3} \cdot \operatorname{Li}\left(\mathrm{Mn}_{0.33} \mathrm{Ni}_{0.33} \mathrm{Co}_{0.33}\right) \mathrm{O}_{2}\right\}(0$ $<\mathrm{x}<1))^{4-6}$ can achieve high reversible capacity $(\sim 200-300 \mathrm{~mA} \cdot \mathrm{h} / \mathrm{g})$ and higher rate capability than other Mn-rich materials due to 3D Li diffusion pathways in the spinel structure. ${ }^{4}$ However, these spinel-layered composite materials undergo severe structural change caused by the Jahn-teller distortion of Mn ions in spinel phase and intrinsic structural instability in layered phase when large amount of Li ions are extracted/inserted resulting in poor energy density and power density. ${ }^{6}$

As a consequence, in these previous approaches, it is difficult for Mn-rich electrode materials to achieve both high energy density and high power density simultaneously, partly because short-range diffusion channels for Li does not allow fast diffusion kinetics in disorderedrocksalt Mn-rich materials, ${ }^{2,3}$ and partly because severe structure distortion in a spinel structure occurs in a wide window of operating potential in spinel-layered composite materials. ${ }^{4-6}$ Here, we report on a new strategy of achieving both high energy density and power density in Mnrich materials by controlling the atoms solubility between the spinel phase and layered phase 
in a composite. Limited atomic solubility in the spinel phase can induce the reaction between these two phases resulting in the defective spinel structure and partially increased cationdisordered layered structure. The resulting Mn-rich material has the highest energy density $1100 \mathrm{~W} \cdot \mathrm{h} / \mathrm{kg}$ and excellent rate capability, up to $10 \mathrm{C}$ rate $(3 \mathrm{~A} / \mathrm{g})$ among reported $\mathrm{Mn}$-rich materials by improving reversible anion redox reaction in the cation-disordered layered structure and facilitating 3D Li diffusion and minimizing structural distortion through the defective spinel structure.

\section{Results}

The quenched (Q) sample forms the reacted spinel-layered composite but the slowlycooled (S) sample does not.

The Mn-rich electrode material that is composed of a spinel-layered composite with nominal composition $0.5 \mathrm{LiNi}_{0.5} \mathrm{Mn}_{1.5} \mathrm{O}_{4}-0.5 \mathrm{Li}_{2} \mathrm{MnO}_{3}\left(=\mathrm{Li}_{1.5} \mathrm{Ni}_{0.25} \mathrm{Mn}_{1.25} \mathrm{O}_{3.5} ; \mathrm{Mn}=83 \%\right.$ of total $\left.\mathrm{TMs}\right)$ was prepared by using a solid-state reaction because of high amount of $\mathrm{Mn}$. To control the atoms solubility between the two phases in the composite, we prepared two samples with different cooling rate after re-annealing at $800{ }^{\circ} \mathrm{C}$; The sample with the quenching process from $800{ }^{\circ} \mathrm{C}$ to room temperature (RT) was prepared and then labelled as a Q sample. As a reference, the sample with a natural cooling process to RT was also prepared and was labelled as a S sample.

Synchrotron XRD diffractions (Fig. 1a) clearly show that both samples are composed of the two phases, a cubic spinel phase (Fm-3m symmetry) such as $\mathrm{LiNi}_{0.5} \mathrm{Mn}_{1.5} \mathrm{O}_{4}$ (LNMO) and a monoclinic layered phase $\left(\mathrm{C} / 2 \mathrm{~m}\right.$ symmetry) such as $\mathrm{Li}_{2} \mathrm{MnO}_{3}$, leading to the formation of spinel-layered composite. ${ }^{4-6}$ Even though the two samples have similar particle size, 
morphology (Fig. 1b and 1c) and actual composition (Supplementary Table. 1), the samples show quite different intensity ratio between the layered phase and the spinel phase in the XRD patterns (Fig. 1a). This indicates that the samples have different interaction between the spinel phase and the layered phase in a composite depending on the cooling rate. Since the $\mathrm{LiNi}_{0.5} \mathrm{Mn}_{1.5} \mathrm{O}_{4}$ (LNMO) spinel phase obtained at temperatures $T>700{ }^{\circ} \mathrm{C}$ exhibits limited solubilities of oxygen and $\mathrm{Ni}$ leading to the formation of the rocksalt $\mathrm{Li}_{x} \mathrm{Ni}_{\mathrm{y}} \mathrm{O}$ phase ${ }^{7-9}$, it appears that thus different cooling rates can strongly affect these solubilities in $\mathrm{LiNi} \mathrm{i}_{0.5} \mathrm{Mn}_{1.5} \mathrm{O}_{4}$ (LNMO) spinel. Even though the quenching process at high temperature $800^{\circ} \mathrm{C}$ to $\mathrm{RT}$ leads easily tyo the formation of the $\mathrm{Li}_{\mathrm{x}} \mathrm{Ni}_{\mathrm{y}} \mathrm{O}$ phase due to limited solubilities, the $\mathrm{Q}$ sample characterized at RT does not have any $\mathrm{Li}_{x} \mathrm{Ni}_{\mathrm{y}} \mathrm{O}$ phase (Fig. 1a). It indicates that the $\mathrm{Li}_{x} \mathrm{Ni}_{\mathrm{y}} \mathrm{O}$ phase at high temperature can be reacted with the $\mathrm{Li}_{2} \mathrm{MnO}_{3}$ layered phase resulting in its disappearance and the formation of the $\mathrm{Ni}$ incorporated $\mathrm{Li}_{2} \mathrm{MnO}_{3}$-like layered phase. As a result, the reaction of $\mathrm{Li}_{\mathrm{x}} \mathrm{Ni} \mathrm{i}_{\mathrm{y}} \mathrm{O}$ phase can increase the intensity ratio of layered/spinel phase in the $\mathrm{Q}$ sample (Fig. 1a). During re-annealing at $800{ }^{\circ} \mathrm{C}$ and then the quenching process to RT, the LNMO spinel can become defective structure due to the loss of the $\mathrm{Li}_{\mathrm{x}} \mathrm{Ni}_{\mathrm{y}} \mathrm{O}$ and limited oxygen solubility at this temperature, and simultaneously the $\mathrm{Li}_{2} \mathrm{MnO}_{3}$ layered phase can have the incorporation of the Ni.

To understand the reaction between the spinel phase and the layered phase in the composite in the samples, neutron powder diffraction (NPD) were carried out (Supplementary Fig. 1 and Supplementary Table 2). NPD Rietveld refinement of phase fractions (Fig. 1d) indicates that the Q sample has a more significant weight fraction of the layered phase ( $45 \mathrm{wt} \%$ ) and less weight fraction of the spinel phase ( $55 \mathrm{wt} \%)$ compared to the S sample, that has $\sim 36 \mathrm{wt} \%$ of the layered phase and $\sim 64 \mathrm{wt} \%$ for the spinel phase, which is similar with the ideal phase fraction obtained from the nominal composition, $0.5 \mathrm{LiNi}_{0.5} \mathrm{Mn}_{1.5} \mathrm{O}_{4}-0.5 \mathrm{Li}_{2} \mathrm{MnO}_{3}$. The increase 
in the phase fraction of the layered phase in the $\mathrm{Q}$ sample indicates that the $\mathrm{Li}_{2} \mathrm{MnO}_{3}$ layered phase can be reacted with the $\mathrm{Li}_{\mathrm{x}} \mathrm{Ni}_{\mathrm{y}} \mathrm{O}$ phase and thus can increase its fraction. Since the two samples have the same overall composition (in Supplementary Table. 1), but different layered/spinel phase fractions (Fig. 1d), it indicates that the two phases in the Q sample have different composition from those in the $\mathrm{S}$ sample. The $\mathrm{S}$ sample has indeed the $\mathrm{LiNi}{ }_{0.5} \mathrm{Mn}_{1.5} \mathrm{O}_{4}$ spinel phase and $\mathrm{Li}_{2} \mathrm{MnO}_{3}$ layered phase, with compositions corresponding to the nominal ones, whereas the Q sample is comprised of a Ni-defective LNMO-like spinel phase and a $\mathrm{Ni}$ containing $\mathrm{Li}_{2} \mathrm{MnO}_{3}$-like layered phase, thus with different compositions from the two phases in the nominal composition.

Refinement results for atomic occupancies of the spinel phase in the samples (Fig. 1e) clearly show that the Q sample has defective LNMO-like spinel phase, whereas the S sample does not have any defects in the LNMO spinel phase (occupancy of the oxygen site is equal to 4). The Q sample has a certain amount of oxygen deficiency, $\sim 0.4$ mol due to limited solubility of oxygen but the S sample does not have (Fig. 1e, left side). Oxygen deficiency in the LNMO spinel is a general phenomenon at $T>700{ }^{\circ} \mathrm{C}{ }^{7,9}$ and thus it can cause the reduction of $\mathrm{Mn}^{4+}$ to $\mathrm{Mn}^{3+}$ leading to the increase in a lattice parameter because $\mathrm{Mn}^{3+}$ ions have larger ionic radius than $\mathrm{Mn}^{4+}$ ions. As a result, the refined lattice parameter of the spinel phase (Supplementary Table. 2) in the Q sample (8.201 $\AA$ ) is much higher than that in the S sample (8.165 $\AA$ ), which is consistent with previous results. ${ }^{7,9,10}$ The oxygen deficiency in the Q sample is expected to yield an increase in the amount of $\mathrm{Mn}^{3+}$ for satisfying the charge balance; this is supported by XANES data of the Q sample (Supplementary Fig. 2). Also the amount of Ni deficiency in the spinel structure due to its limited solubility at high temperature is refined with neutron powder diffraction; $0.1 \mathrm{~mol}$ of $\mathrm{Ni}$ in the 16d sites of the spinel phase in the Q sample is vacant (Fig. 1e, right side) leading to the formation of defective LNMO-like spinel. This deficient amount 
of $\mathrm{Ni}$ in $\mathrm{LNMO}$ spinel can be incorporated into the $\mathrm{Li}_{2} \mathrm{MnO}_{3}$ phase at high temperature $(T=\sim$ $800{ }^{\circ} \mathrm{C}$ ) via the reaction of the $\mathrm{Li}_{x} \mathrm{Ni}_{\mathrm{y}} \mathrm{O}$ phase. This reaction leads to the formation of the $\mathrm{Ni}$ incorporated $\mathrm{Li}_{2} \mathrm{MnO}_{3}$-like phase in the $\mathrm{Q}$ sample (Fig. 1e, right side) leading to the increase in the phase fraction of the layered phase compared to the S sample with ideal nominal composition (Fig. 1d). The incorporated $\mathrm{Ni}$ in the $\mathrm{Li}_{2} \mathrm{MnO}_{3}$-like phase can sit in both Li layers ( $0.08 \mathrm{~mol}$ ) and TM layers ( $0.02 \mathrm{~mol}$ ) (Supplementary Table. 2$)$. Especially, the amount of $\mathrm{Ni}$ in Li layers ( $0.08 \mathrm{~mol})$ in a layered structure can increase the degree of the cation disordering. After quenching from high temperature $\left(\sim 800^{\circ} \mathrm{C}\right)$ to room temperature, the $\mathrm{Q}$ sample exhibits a quite different composite structure from the $\mathrm{S}$ sample due to the reaction of the $\mathrm{Li}_{x} \mathrm{Ni}_{y} \mathrm{O}$ phase with the layered $\mathrm{Li}_{2} \mathrm{MnO}_{3}$ phase that is originated from limited atomic solubilities in the spinel. The reaction between the two layered and spinel phases in a composite at high temperature results in the change in their respective compositions yielding an increase (decrease) in the phase fraction of the $\mathrm{Li}_{2} \mathrm{MnO}_{3}$-like layered phase (the LNMO-like spinel phase) in the Q sample. As a result, the Q sample can form a composite with defective LNMOlike spinel phase, containing an increase amount of $\mathrm{Mn}^{3+}$ ions, Ni vacancies, and the oxygen deficiency, and $\mathrm{Ni}$ incorporated $\mathrm{Li}_{2} \mathrm{MnO}_{3}$-like layered phase. On the opposite, the S sample (Fig. 1d) does not have any of these features in the two phases in the composite.

To figure out how the changed composite structure in the Q sample substantially affects local Li environments in both spinel and layered phases, ${ }^{7}$ Li Magic Angle Spinning (MAS) Nuclear Magnetic Resonance (NMR) measurements were carried out on Q and S samples. Figure 1f displays only isotropic resonances deduced from the experimental spectra simulations (for experimental spectra, see supporting onformation Fig. X) Careful déconvolution of both spectra obtained for Q and S samples clearly indicates that different local environments of Li in the spinel and layered structure are distinguishable: ${ }^{11,12}$ two resonances at $\sim 700 \mathrm{ppm}$ and 
1700 ppm can be assigned to the typical octahedral Li local environments in the layered structure. The other resonances between 1100 and 1300 ppm can be attributed to tetrahedral Li local environments in the spinel structure (Fig. 1f and Supplementary Fig. 3). Fitted ${ }^{7}$ Li MAS NMR results show that the spectrum corresponding to the Q sample displays quite different hyperfine shifts (Fig. 1f) and apparent linewidth ( $\Delta \delta$ in Fig. 1g) for both spinel and layered environments compared to the S sample (Supplementary Table 3).

The observed hyperfine shift (“O” in Fig. 1f) and apparent linewidth ( $\Delta \delta$ in Fig. 1g) of ${ }^{7} \mathrm{Li}$ MAS NMR signal for Li local environments in the structure of paramagnetic materials such as the spinel structure, depend typically on different degree of $\mathrm{Ni} / \mathrm{Mn}$ ordering/distribution in the vicinity as well as the average oxidation state of Mn ions. ${ }^{13,14}$ The signals assigned to Li spinellike local environment (between 110 and 1300 ppm) exhibit both different hyperfine shifts and apparent linewidths. Although the signal of tetrahedral Li in the Q sample could be fitted with one symmetric resonance while the more pronounced asymmetric shape of the tetrahedral $\mathrm{Li}$ in the case of the S sample required a fit with 2 additional resonances between 1285 and 1300 ppm, both can be considered as the sum of overlapping resonances corresponding to slightly different local environments in terms of $\mathrm{Ni} / \mathrm{Mn}$ distribution. The difference between signals observed for the Q and S sample suggests thus that the LNMO-like spinel phase in the Q sample has different degree of $\mathrm{Ni} / \mathrm{Mn}$ ordering and oxidation state of $\mathrm{Mn}$ ions from the LNMO spinel phase in the $\mathrm{S}$ sample. In particular, the $\mathrm{Q}$ sample does not have the two low intensity resonances with a higher shift above 1200 ppm but the S sample does clearly contain these specific environments (black arrows in Fig. 1f). Considering that $\mathrm{Mn}^{4+}$ ions in the first coordination sphere of a tetrahedral Li ion in a LNMO spinel structure lead to higher shifts than $\mathrm{Mn}^{3+}$ ions, the negligible intensity of resonances at $>1200 \mathrm{ppm}$ in the Q sample can be attributed to an overall increase in the amount of $\mathrm{Mn}^{3+}$ ions, whereas the resonances with higher shifts (between 1285 and $1300 \mathrm{ppm}$ ) in the S sample can be attributed to $\mathrm{Mn}^{4+}$ ions-rich 
environments. ${ }^{13,14}$ This observation of increased amount of $\mathrm{Mn}^{3+}$ ions in the LNMO-like phase of the Q sample is consistent with the refinement of the lattice parameter (Supplementary Table 2) and XANES data (Supplementary Fig. 2). Secondly, concerning the remaining major resonance at $1100 \mathrm{ppm}$ ( $\mathrm{Li}$ local environments in the LNMO-like spinel phase), the spectrum of Q sample displays a much broader linewidth $(\Delta \delta)$ than that of the $\mathrm{S}$ sample (Fig. 1g). In the LNMO spinel structure, Li in tetrahedral sites has 12 TMs (ideally $9 \mathrm{Mn}$ and $3 \mathrm{Ni}$ ) as first nearest neighbors, so the change in the ratio of $\mathrm{Ni} / \mathrm{Mn}$ or the degree of $\mathrm{Ni} / \mathrm{Mn}$ ordering between the two TM sites can lead to an increased NMR shift distribution and therefore an increased apparent linewidth. ${ }^{13,14}$ The presence of Ni vacancies may also increase the NMR shift distribution as well as introduce additional site distortions in the vicinity of the observed Li nucleaus, also resulting in an increased apparent linewidth. ${ }^{7} \mathrm{Li}$ NMR measurements suggest that the LNMO-like spinel phase in the Q sample has an overall higher amount of $\mathrm{Mn}^{3+}$ ions as well as an increased amount of $\mathrm{Ni}$ vacancies or degree of $\mathrm{Ni} / \mathrm{Mn}$ disordering with respect to the LNMO spinel in the S sample.

In an ideal $\mathrm{Li}_{2} \mathrm{MnO}_{3}$ layered structure, all octahedral $\mathrm{Li}$ ions are surrounded by $\mathrm{Mn}^{4+}$ ions and thereby give rise to higher hyperfine shifts compared to those observed typically in layered structures that contain $\mathrm{Ni}$ atoms. ${ }^{11}$ Here, the resonances attributed to octahedral Li local environments in the $\mathrm{Li}_{2} \mathrm{MnO}_{3}$-like layered phase in the $\mathrm{Q}$ sample show as expected, lower hyperfine shifts ( $1760 \mathrm{ppm}$ and $~ 632 \mathrm{ppm}$ ) (Fig. 1f) compared to the $\mathrm{Li}_{2} \mathrm{MnO}_{3}$ layered phase in the S sample ( 1798 ppm and $700 \mathrm{ppm}$ ) (Fig. 1g). The lowering of the overall hyperfine shifts in the Q sample is attributed to incorporation of $\mathrm{Ni}$ into the $\mathrm{Li}_{2} \mathrm{MnO}_{3}$ layered phase. We attribute here this incorporation process to the reaction with the $\mathrm{Li}_{x} \mathrm{Ni}_{\mathrm{y}} \mathrm{O}$ phase, caused by its limited solubility in the LNMO spinel at high temperature. The much broader apparent linewidth $(\Delta \delta)$ observed for the $\mathrm{Li}$ resonances in the $\mathrm{Li}_{2} \mathrm{MnO}_{3}$-like layered phase in the $\mathrm{Q}$ 
sample, in particular for environments corresponding to $\mathrm{Li}$ in Li layers (Fig. 1g) is related to a wider distribution of $\mathrm{Li}$ local environments due to the presence of $\mathrm{Ni}$ in its vicinity over different possible octahedral sites (both in Li layers and TM layers) of the layered structure, creating various $\mathrm{Li}$ local environments with different numbers of $\mathrm{Mn}$ and $\mathrm{Ni}$ in the first and second coordination cationic sphere (Supplementary Table. 3). ${ }^{12,15}$ The resulting overlapping resonances could not be resolved in the ${ }^{7} \mathrm{Li}$ MAS NMR spectra. As a result, the increased apparent broadness indicates that $\mathrm{Li}$ environments in the Q sample deviate strongly from the ideal $\mathrm{Li}_{2} \mathrm{MnO}_{3}$ due to the incorporation of $\mathrm{Ni}$ in both the $\mathrm{Li}$ layer or $\mathrm{TM}$ layer of $\mathrm{Li}_{2} \mathrm{MnO}_{3}$-like layered phase. Neutron and NMR measurements suggest therefore that the $\mathrm{Q}$ sample is composed of (1) a defective LNMO-like spinel phase that has an increased amount of $\mathrm{Mn}^{3+}$ ions from oxygen deficiency and a higher the degree of Ni/Mn disordering with Ni vacancies, and (2) a $\mathrm{Li}_{2} \mathrm{MnO}_{3}$-like layered phase that incorporates $\mathrm{Ni}$, which can increase partial cation disordering.

\section{TEM measurements confirm that the $Q$ sample has defective spinel - partially cation} disordered layered composite but the S sample does not.

To further characterize the local structures of the spinel phase and the layered phase in the

samples, Scanning Transmission Electron Microscopy (STEM) measurements were carried out. High Angle Annular Dark Field (HAADF)-STEM image (Fig. 2a) along with the [110] direction of the spinel phase and its corresponding intensity profile in the $\mathrm{Q}$ sample clearly show lower intensity in each TM site (16d) compared to the ideal spinel structure. ${ }^{16}$ In Fig. $2 b$, a (1)-line profile from Fig. 2a shows an intensity ratio between the two different $16 \mathrm{~d}$ sites much lower than 0.5 (orange arrows). Generally, the intensity ratio between the two TM sites (16d) 
in ideal Li-TM-O spinel structure is 0.5 indicating that the intensity of TM1 in 16d site is about half of that at the TM2 in $16 \mathrm{~d}$ site. ${ }^{16} \mathrm{~A}$ (2)-line profile (in Fig. 2b) from Fig. 2a also shows lower intensity peaks (orange arrows) among the each TM sites (16d), which is clearly different to the ideal spinel structure that should have the same intensity of the peaks among the each TM site along the (2)-line (atomic configuration in ideal spinel in Fig. $2 a) .{ }^{16}$ These HAADF images indicate that the Q sample has certain amount of Ni vacancies at $16 \mathrm{~d}$ sites in the LNMO spinel. Furthermore, Annular Bright Field (ABF)-STEM image (Fig. 2c), which can visualize light elements such as Li and oxygen, ${ }^{16,17}$ along with the [110] direction of the LNMO spinel phase and its corresponding intensity profiles clearly show that the Q sample can have the deficiency in cations (e.g., $\mathrm{Li}$ or $\mathrm{Ni}$ ) and $\mathrm{O}$ atoms. Compared to the (1)-line profile, which does not have any defects, a (2)-line profile (orange arrows in Fig. 2d) clearly shows the deficiency in cations (e.g., $\mathrm{Li}$ or $\mathrm{Ni}$ ) and $\mathrm{O}$ atoms. These STEM results clearly demonstrate that the $\mathrm{Q}$ sample contains a defective LNMO-like spinel phase, which has some oxygen vacancies and some deficiency of cations (e.g., Li or $\mathrm{Ni}$ ) due to limited solubilities of the $\mathrm{Ni}$ and oxygen in the spinel at high temperature. ${ }^{7,9}$ Also, the defective LNMO-like spinel phase in the Q sample has a higher degree of $\mathrm{Ni} / \mathrm{Mn}$ disordering as observed by the weak additional spots in diffraction pattern along the [110] zone-axis (Supplementary Fig. 4b) compared to the LNMO spinel phase in the S sample along the [112] zone-axis (Supplementary Fig. 4e); these additional spots are a result of the degree of $\mathrm{Ni} / \mathrm{Mn}$ ordering at octahedral $4 \mathrm{~b}$ site for $\mathrm{Ni}$ and octahedral 12c site for Mn in the ordered LNMO spinel structure. ${ }^{14,18}$

In the $\mathrm{Li}_{2} \mathrm{MnO}_{3}$-like layered phase in the Q sample, HAADF-STEM images (Fig. 2e) along with the [110] direction of the layered phase show the incorporation of $\mathrm{Ni}$ in the $\mathrm{Li}_{2} \mathrm{MnO}_{3}-$ like layered phase. Considering that HAADF-STEM images only visualize heavy elements such as $\mathrm{Ni}$ and Mn whereas light elements like Li cannot be directly observed, ${ }^{17,19,20}$ HAADF image 
along with the [110] direction in the $\mathrm{Li}_{2} \mathrm{MnO}_{3}$-like layered phase in the $\mathrm{Q}$ sample (Fig. 2e) shows that the Li layer (2)-line in Fig. 2e) displays a strong contrast compared to the negligible contrast in ideal $\mathrm{Li}_{2} \mathrm{MnO}_{3}$ structure due to the only presence of $\mathrm{Li}$ atoms. This result clearly indicates the presence of heavy elements such as $\mathrm{Ni}$ in Li layer. As a results, the TM layer (1)line in Fig. 2e) has a few additional atoms instead of Li (orange arrow in Fig. 2f) compared to an ideal Li-Mn-Mn like arrangements in the ideal $\mathrm{Li}_{2} \mathrm{MnO}_{3}$ structure. ${ }^{19,20}$ The corresponding intensity profiles (Fig. 2f) also show lots of Ni atoms in the Li-layer (cation disordering, (2)line in Fig. 2f), and a few additional Ni atoms in TM-layers (orange arrows, (1)-line in Fig. 2f) in the $\mathrm{Li}_{2} \mathrm{MnO}_{3}$-like layered phase, which are consistent with the refined $\mathrm{Ni}$ occupancy in the $\mathrm{Li}_{2} \mathrm{MnO}_{3}$-like layered phase of Q sample (Supplementary Table. 2). However, the S sample does not show any additional atoms in both $\mathrm{Li}$ layer and $\mathrm{TM}$ layer in the $\mathrm{Li}_{2} \mathrm{MnO}_{3}$ phase (Supplementary Fig. 4h, i) indicating that there is a negligible cation disordering in the $\mathrm{Li}_{2} \mathrm{MnO}_{3}$ layered structure in the S sample (Supplementary Table. 2).

Schematic diagrams (Fig. 2g) of the Q and S samples represent different structural/chemical characteristics of the composite in the two samples even though they both have the same nominal composition and similar particle size and morphology. The Q sample is composed of the defective LNMO-like spinel phase with deficient oxygen and Ni with the higher degree of $\mathrm{Ni} / \mathrm{Mn}$ disordering, and the partially cation-disordered $\mathrm{Li}_{2} \mathrm{MnO}_{3}$-like layered phase via incorporation of $\mathrm{Ni}$, especially lot of $\mathrm{Ni}$ in $\mathrm{Li}$ layer. This reacted composite in the Q sample is formed due to the limited atomic solubilities in LNMO spinel at $T>700{ }^{\circ} \mathrm{C}^{7,9}$ that can induce the reaction of the $\mathrm{Li}_{x} \mathrm{Ni}_{y} \mathrm{O}$ phase with the $\mathrm{Li}_{2} \mathrm{MnO}_{3}$ layered phase at high temperature. In contrast, the S sample is a typical spinel-layered composite, which is composed of the typical LNMO spinel phase with $\mathrm{Ni} / \mathrm{Mn}$ ordering with negligible oxygen and Ni vacancies, ${ }^{7,9}$ and the typical $\mathrm{Li}_{2} \mathrm{MnO}_{3}$ layered phase with negligible cation-disordering. 


\section{Different composite structures in the samples show different electrochemical properties;}

The unexpectedly high reversible capacity of $\sim 340 \mathrm{~mA} \cdot \mathrm{h} / \mathrm{g}$ is achieved in the $\mathrm{Q}$ sample.

The different and particular composite structure characterizing the Q sample compared to the S sample leads to significantly improved electrochemical activities (Fig. 3a). The Q sample can achieve high reversible capacity, $340 \mathrm{~mA} \cdot \mathrm{h} / \mathrm{g}$ with high energy density $\sim 1100 \mathrm{~W} \cdot \mathrm{h} / \mathrm{kg}$ (at $6.0 \mathrm{~mA} / \mathrm{g}$, Galvanostatic test), whereas the S sample can achieve only the capacity of $\sim 280$ $\mathrm{mA} \cdot \mathrm{h} / \mathrm{g}$ with low energy density $\sim 820 \mathrm{~W} \cdot \mathrm{h} / \mathrm{kg}$ (at $6.0 \mathrm{~mA} / \mathrm{g}$ ) in the voltage range from $2.0 \mathrm{~V}$ to $4.9 \mathrm{~V}$ at RT. To understand the reason why the Q sample has much higher reversible capacity than the S sample, redox reactions in the samples were identified by ploting the derivative of capacity with respect to voltage $(\mathrm{d} Q / \mathrm{d} V)$.

In both samples, the spinel and layered phases have distinct electrochemical characteristics (Fig. 3b-d). The redox reactions in the spinel phase cause five voltage plateaus (three in region (3), two in region (1), in Fig. 3c-d). In region (3), the two plateaus at $\sim 4.7 \mathrm{~V}$ can originate from the $\mathrm{Ni}^{2+} / \mathrm{Ni}^{4+}$ redox reaction, and the plateau at $\sim 4.0 \mathrm{~V}$ can originate from the $\mathrm{Mn}^{3+} / \mathrm{Mn}^{4+}$ redox reaction. ${ }^{10,14}$ In this region, the $\mathrm{Q}$ sample has a redox reaction at $\sim 4.0 \mathrm{~V}$ involving a higher capacity compared to the corresponding redox reaction at $\sim 4.7 \mathrm{~V}$ for the $\mathrm{S}$ sample. The increased redox reaction at $\sim 4.0 \mathrm{~V}$ in the $\mathrm{Q}$ sample can be a result of the increase in the amount of $\mathrm{Mn}^{3+}$ ions as shown in XANES data (Supplementary Fig. 2). However, the decreased redox reaction at $\sim 4.7 \mathrm{~V}$ in the $\mathrm{Q}$ sample can be a result of the decrease in the $\mathrm{Ni}^{2+} / \mathrm{Ni}^{4+}$ redox couple

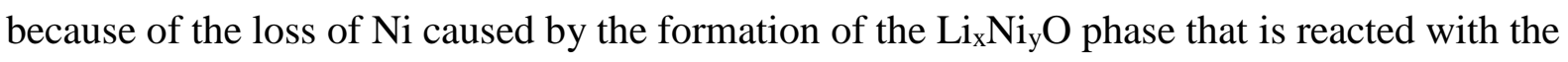
layered phase at high temperature.

In region (1), the plateaus at $\sim 2.7 \mathrm{~V}$ and $\sim 2.1 \mathrm{~V}$ can originate from the $\mathrm{Mn}^{3+} / \mathrm{Mn}^{4+}$ redox 
reaction associated with the insertion of $\mathrm{Li}$ ions into the empty 16c octahedral sites in the cubic spinel structure, or extraction of $\mathrm{Li}$ ions from them; the insertion of $\mathrm{Li}$ is accompanied by a phase transition from cubic to tetragonal spinel phase that can induce Jahn-Teller distortion. ${ }^{21-}$ ${ }^{23}$ The differences in the plateau length at $\sim 2.7 \mathrm{~V}$ and $\sim 2.1 \mathrm{~V}$ generally originate from the degree of Ni/Mn ordering in LNMO spinel; ${ }^{21}$ As the Ni/Mn ordering increases, the plateau at $\sim 2.7 \mathrm{~V}$ is lengthened, and as the $\mathrm{Ni} / \mathrm{Mn}$ ordering decreases, the plateau at $\sim 2.1 \mathrm{~V}$ is lengthened. The Q sample shows much longer plateau at $\sim 2.1 \mathrm{~V}$ than at $\sim 2.7 \mathrm{~V}$, whereas the $\mathrm{S}$ sample has much longer $\sim 2.7 \mathrm{~V}$ plateau than $\sim 2.1 \mathrm{~V}$ plateau, supporting a higher $\mathrm{Ni} / \mathrm{Mn}$ disordering in the spinel phase of the Q sample with respect to the $S$ sample. This is consistent with the TEM diffraction data (Supplementary Fig. 4b, e). Even if the phase fraction of the spinel phase in the S sample (63\% in Fig. 1d) is a little higher than that in the Q sample (55\% in Fig. 1d), the capacity below $3 \mathrm{~V}$ is similar in both Q sample $(\sim 120 \mathrm{~mA} \cdot \mathrm{h} / \mathrm{g})$ and S sample $(\sim 125 \mathrm{~mA} \cdot \mathrm{h} / \mathrm{g})$. Taking into the phase fraction of the spinel (Fig. 1d), the lower capacity below 3V in the S sample than the Q sample is related with severe structure distortion via phase transition from cubic to tetragonal spinel, which can make the accommodation of a lot of $\mathrm{Li}$ in empty 16c sites difficult. $^{21}$

However, the unexpectedly high reversible capacity of $~ 340 \mathrm{~mA} \cdot \mathrm{h} / \mathrm{g}$ in the Q sample cannot be explained by redox reactions only from the spinel phase in regions (1) and (3), because both samples have similar capacity contribution from the spinel phase. Considering the different voltage profiles of the Q-sample and the S-sample (Fig. 3a), the layered phases in the two samples have quite different electrochemical activities and redox reactions in regions (2) and (3). In typical $\mathrm{Li}_{2} \mathrm{MnO}_{3}$ layered phase, the voltage plateau at $\sim 4.5 \mathrm{~V}$ in region (3) during the $1^{\text {st }}$ charge process is related to the oxidation of oxygen and the extraction of Li ions from the lattice; this plateau is directly linked to the electrochemical activation of the $\mathrm{Li}_{2} \mathrm{MnO}_{3}$ phase. ${ }^{24-26}$ 
The plateau at $4.5 \mathrm{~V}$ is significantly longer for the Q sample than for the S sample (Fig. 3a); this different length of the plateau indicates that the oxygen redox reaction in the layered phase $^{25,26}$ in the Q sample has a much higher electrochemical activity than in the S sample. During the $1^{\text {st }}$ discharge, the voltage profile between $4.0 \mathrm{~V}$ and $3.0 \mathrm{~V}$ in region (2) shows a sloping process, which is a result of the insertion of $\mathrm{Li}$ and the corresponding of the oxygen reduction reaction in the layered phase. ${ }^{25,26}$ Surprisingly, the Q sample shows a significantly increased reduction peak of the oxygen redox reaction (Fig. 3c), whereas the S sample shows negligible reduction peak (Fig. 3d). The different electrochemical activity in the discharge in the two samples is consistent with the different electrochemical activation of the layered phase during the voltage plateau at $4.5 \mathrm{~V}$ during the $1^{\text {st }}$ charge, where the oxygen redox reactions mainly occur. ${ }^{25,26}$ This voltage feature during the $1^{\text {st }}$ charge process is similar to that in conventional Li-rich layered materials that have active oxygen redox reaction. ${ }^{25,26}$ Increased oxygen redox reaction is further evidenced by pre-edge region below $\sim 534 \mathrm{eV}$ in the $\mathrm{O}$ K-edge SXAS (Soft X-ray Absorption Spectroscopy) spectra in Supplementary Fig. 5. This region can be attributed to transitions from the $\mathrm{O} 1 \mathrm{~s}$ to the unoccupied state in $\mathrm{O} 2 \mathrm{p}$ orbital, and to transitions from the $\mathrm{O} 1 \mathrm{~s}$ to the empty hybridized TM d - O 2p orbitals. ${ }^{27,28}$ The integrated intensity of the $\mathrm{O}$ K-edge in the pre-edge region between 4.0 V and 3.0 V increases even though $\mathrm{Ni}$ and Mn K-edge are not significantly shifted to lower energy states in this region. This indicates that the main redox reaction is most probably the oxygen redox reaction rather than TM redox reactions and thereby the oxygen redox reaction mainly contributes to the obtained capacity. As a result, the significantly increased oxygen redox activity in the $\mathrm{Li}_{2} \mathrm{MnO}_{3}$-like layered phase in the Q sample leads to much higher capacity than the S sample. Furthermore, this increased electrochemical activity of the $\mathrm{Li}_{2} \mathrm{MnO}_{3}$-like layered phase in the Q sample leads to the increase in the energy density (Fig. 3b), which is obtained by the integration of voltage and capacity curve in region (2) between $4.0 \mathrm{~V}$ and 3.0 V. High electrochemical activity in the 
Q sample demonstrates that the incorporation of $\mathrm{Ni}$ into the $\mathrm{Li}_{2} \mathrm{MnO}_{3}$-like layered phase in the Q sample can help oxygen redox activity of the $\mathrm{Li}_{2} \mathrm{MnO}_{3}$ layered phase to be enhanced. ${ }^{29,30}$

The highest energy density and superior power density are simultaneously achieved in the Q sample compared to other Mn-rich electrode materials

In addition to improved energy density in the Q sample, the different composite structure in the Q sample leads to superior electrochemical performance compared to the S sample. The Q sample achieved significantly improved discharge capacity and rate capability compared to the S sample and previously reported Mn-rich electrode materials. The Q sample shows high discharge capacities of $>300 \mathrm{~mA} \cdot \mathrm{h} / \mathrm{g}$ for subsequent cycles (Fig. 4a) with $\sim 3.3 \mathrm{~V}$ average discharge potential, which is determined by the voltage at half of the obtained capacity; this indicates that substantially activated redox reactions for both TM redox and O redox in the Q sample can be reversible for cycles. This result in the case of the Q sample can be originated from the different composite structure that can lead to a resilient layered and spinel structure even after an extended cycling. Compared to all reported Mn-rich materials including spinellayered composite materials including the S sample, ${ }^{4,5,31}$ the $\mathrm{Q}$ sample shows much higher reversible capacity for cycles. The remarkably high energy density of the Q sample is the highest yet achieved in materials in which $>80 \%$ of the TMs are Mn (Fig. $4 \mathrm{~b}){ }^{2-6}$

The Q sample also showed remarkably improved rate capability and high power density (Fig. 4c-f). A rate of $n \cdot C$ corresponds to a full discharge in $1 / n$ hours. The $Q$ sample was charged at $\mathrm{C} / 12$ rate $(1 \mathrm{C}$ rate $=300 \mathrm{~mA} / \mathrm{g})$ and discharged at various rates. It achieves $\sim 200 \mathrm{~mA} \cdot \mathrm{h} / \mathrm{g}$ of capacity at $6 \mathrm{C}$ discharge rate $(1,800 \mathrm{~mA} / \mathrm{g}), \sim 180 \mathrm{~mA} \cdot \mathrm{h} / \mathrm{g}$ at $8 \mathrm{C}$ discharge rate $(2,400 \mathrm{~mA} / \mathrm{g})$, and reasonable capacity of $\sim 160 \mathrm{~mA} \cdot \mathrm{h} / \mathrm{g}$ even at 10C discharge rate $(3,000 \mathrm{~mA} / \mathrm{g}, 6 \mathrm{~min}$ 
discharge) (Fig. 4c) with no severe capacity fading at these high rates for $\sim 10$ cycles (Fig. 4d), whereas the other Mn-based materials show no reasonable capacity above $3 \mathrm{~A} / \mathrm{g} \cdot{ }^{2-4}$ Interestingly, reasonable capacities at high rates (above 6C) can be obtained below $3.0 \mathrm{~V}$; At $6 \mathrm{C}$ rate, $\sim 50 \%$ of the obtained capacity is from this voltage region. Considering that high voltage polarization, $\sim 0.5 \mathrm{~V} \sim 0.6 \mathrm{~V}$ can occur at these high rates, in this voltage range, the main redox reactions are probably the oxygen redox reaction in the $\mathrm{Li}_{2} \mathrm{MnO}_{3}$ layered phase, ${ }^{20,25,26}$ which was believed to be associated with sluggish $\mathrm{Li}^{+}$diffusion kinetics, ${ }^{20}$ and $\mathrm{Mn}$ redox reaction in the LNMO spinel phase that can occur via additional Li insertion in octahedral empty $16 \mathrm{c}$ sites below $3 \mathrm{~V}$, which can induce severe Jahn-Teller distortion caused by a phase transformation from cubic to tetragonal spinel phase. ${ }^{21,22,32,33}$ This reasonable contribution below 3.0 $\mathrm{V}$ at high rates in the Q sample indicates an improved kinetics of the spinel phase in the Q sample because the redox reaction below $3.5 \mathrm{~V}$ is strongly limited by the phase transition from cubic phase to tetragonal phase in the spinel structure, especially at high rates. ${ }^{6,21,22,32}$ Furthermore, the Q sample in the 2C charge (600 mA/g) - 2C discharge test and 1C-1C test (Supplementary Fig. 6a, c) shows reasonable capacity of $\sim 170 \mathrm{~mA} \cdot \mathrm{h} / \mathrm{g}$ and $\sim 210 \mathrm{~mA} \cdot \mathrm{h} / \mathrm{g}$, which capacity mainly comes from reactions at $<3.5 \mathrm{~V}$. However, the S sample shows much lower capacity of $\sim 100 \mathrm{~mA} \cdot \mathrm{h} / \mathrm{g}$ at $1 \mathrm{C}-1 \mathrm{C}$ test (Supplementary Fig. 6b) than the Q sample, which results in low capacity contribution at $<3.5 \mathrm{~V}$.

This can be explained by a strong alteration of the redox reaction $(<3.5 \mathrm{~V})$ in typical LNMO spinel phase in the S sample since it undergoes severe structural change caused by the Jahnteller distortion of Mn ions leading to higher kinetic barrier in spinel-layered composite. ${ }^{6,21,22,32}$ Therefore, this result can indicate that superior power density and high discharge capacity in the Q sample can stem from defective structural features in the LNMO-like spinel phase. This highest power density in a Co-free Mn-rich electrode material of the Q sample (Fig. 4e) appears 
to be kinetically comparable to other TM-based materials, contrary to previous observations in Mn-based electrode materials. ${ }^{2-6}$

\section{Different composite structure in the two phases in the $Q$ sample leads to the suppression of severe structural changes during lot of $\mathrm{Li}$ extraction/insertion.}

The particular composite framework of the Q sample, comprised of defective spinel domains along with partially cation-disordered layered domains substantially affects the overall structure stability of the composite upon operating in a wide electrochemical potential window (from 2.0 to $4.9 \mathrm{~V}$ ) and can yield high reversible energy density (Fig. 4b) and excellent power density (Fig. 4e). To understand these remarkable improved electrochemical performance in the Mn-rich material, voltage curves and the structural evolution of the S sample (Fig. 5a) and the Q sample (Fig. 5b) were studied with synchrotron X-ray (SXRD) diffraction during two cycles. The intensity of ( $\left(\begin{array}{lll}1 & 1 & 1\end{array}\right)$ peak of the cubic spinel phase and $\left(\begin{array}{lll}0 & 0 & 3\end{array}\right)$ peak of the layered phase at $\sim 18^{\circ}$ in both samples were separated to higher and lower angles, respectively. This separation is consistent with the reported results in literature. ${ }^{21,34}$ After the $1^{\text {st }}$ charge process, the $\left(\begin{array}{lll}0 & 0 & 3\end{array}\right)$ peak of the layered phase decreased sharply and shifted to lower angle in the Q sample (Fig. 5b), but negligibly changes in the S sample (Fig. 5a). This different behavior indicates that the layered phase in the $\mathrm{Q}$ sample undergoes a much more significant electrochemical reaction than that in the S sample. This result is consistent with the higher charge capacity of the $\mathrm{Q}$ sample associated with the long voltage plateau at $\sim 4.5 \mathrm{~V}$ in $1^{\text {st }}$ charge process compared to the S sample (Fig. 3a). After $1^{\text {st }}$ discharge to $2.0 \mathrm{~V}$, the diffraction pattern of the S sample displays new peaks corresponding to the tetragonal spinel phase in addition to those assigned to the layered phase but does not have any peaks that can be attributed to the cubic phase (yellow shaded region in Fig. 5a). This observation indicates that the cubic spinel 
phase in the S sample is completely transformed to the tetragonal one below $3 \mathrm{~V}$ at the end of the discharge. In contrast, in the Q sample, the tetragonal spinel phase and the layered phase coexist and unexpected large portion of the cubic spinel phase is still present even after $1^{\text {st }}$ discharge (yellow shaded region in Fig. 5b). The existence of the cubic spinel phase in the Q sample at the end of discharge can be originated from either incomplete phase transformation, which can cause lower capacity, or the stabilization of the cubic phase, which can mitigate severe structure change involved in the tetragonal phase transformation. Since the Q sample has similar capacity with the S sample below 3V despite less spinel phase fraction in the Q sample than in the S sample (Fig. 1d), the existence of the cubic phase in the Q sample after $1^{\text {st }}$ discharge indicates the stabilization of the cubic phase even with large amount of Li insertion rather than incomplete phase transformation. After $2^{\text {nd }}$ discharge process, the S sample shows still low electrochemical activity for both redox reactions of the layered phase ( $3 \sim 4 \mathrm{~V})$ and the spinel phase (below 3V). Especially, residual cubic spinel phase in the S sample after $2{ }^{\text {nd }}$ cycles (Fig. 5a) indicates that the phase transition from cubic to tetragonal spinel is incomplete with still low capacity below 3V partly due to involved large structure distortion that can make accommodation of a lot of Li in empty 16 c sites difficult. ${ }^{21}$ In contrast, in the Q sample after $2^{\text {nd }}$ discharge process, the ratio of tetragonal spinel phase has decreased while the ratio of cubic phase has sharply increased with the presence of the layered phase. Since the capacity obtained at the $2^{\text {nd }}$ discharge is similar to that of $1^{\text {st }}$ discharge in the $\mathrm{Q}$ sample with full capacity below $3 \mathrm{~V}$, the increase in the cubic spinel phase after $2^{\text {nd }}$ discharge compared to after $1^{\text {st }}$ discharge can indicate a further stabilization of the cubic phase rather than an incomplete phase transformation. The stabilization of the cubic spinel phase below 3V in the Q sample seems then to suppress subsequently the phase transition from the cubic to the tetragonal phase that causes usually severe structural distortion. As a result, this mitigation of the structural change can substantially improve the kinetics of the Q sample. 
To further understand the structural evolution of the samples were studied with synchrotron X-ray diffraction (SXRD) by using Le Bail refinements allowing the determination of lattice parameters in the co-existence of multiple phases (Supplementary Fig. 7-8; Supplementary Table 4). ${ }^{35-37}$ Given that the important qualitative estimates can be derived from the position and relative intensity of peaks, in particular the Le Bail refinement is crucially useful when attempting to distinguish between competing structural models. ${ }^{35-37}$ The change in the lattice parameters and the volume upon cycling for two cycles were obtained by Le bail refinement in the S sample and the Q sample (Fig. 5c and Supplementary Table 4).

To compare the lattice parameters after cycling, the lattice parameters of the tetragonal spinel phase ( $a_{\mathrm{t}}$ and $\left.c_{\mathrm{t}}\right)$ are expressed in terms of the lattice parameter $\left(a_{\mathrm{c}}\right)$ of the cubic phase by using following relations; $a_{\mathrm{t}}=a_{\mathrm{c}} / \sqrt{ } 2$ and $c_{\mathrm{t}}=a_{\mathrm{c} .}{ }^{21}$ As a result, in the Q sample, the volume of the spinel phase during 2 cycles is increased by $6.61 \%$ during the phase transition from cubic to tetragonal phase; this is much smaller than the volume increase of the spinel in the S sample (7.50\%) and reported LNMO spinel (11.5\%) $)^{21}$ even with almost full insertion/extraction of Li in a wide operating potential window (2.0 to $4.9 \mathrm{~V}$ ) (Supplementary Table 4). These smaller volume change in the Q sample compared to the S sample can be ascribed to defective structural features of the Q sample that can change the size of empty 16c sites ${ }^{21}$ and thereby can stabilize the cubic spinel phase. Rietveld refinement results of neutron diffraction measurements in the pristine materials (Fig. 5d) clearly show that the Q sample has much higher average polyhedral volume $\left(\sim 13.52 \AA^{3}\right)$ of empty $16 c$ sites with increasing distance between oxygen ions of empty 16c site (polyhedral in Fig. 5d) in cubic spinel than that of S sample $\left(\sim 13.25 \AA^{3}\right)$. It indicates that defective structural features of the spinel phase in the Q sample such as Ni vacancies in $16 \mathrm{~d}$ sites, oxygen vacancies, and $\mathrm{Ni} / \mathrm{Mn}$ disordering can increase the size of empty 16c sites. The enlargement of the 16c sites caused by these defective structural features in the spinel 
phase can mitigate severe structural changes induced by the phase transition to the tetragonal spinel phase below 3V region in the Q sample even after insertion of large amount of Li leading to high reversible energy density and superior power capability even with a wide operating voltage range in the Q sample.

Also, the change in the $c / a$ ratio of the layered phase during two cycles (Supplementary Table. 4) is smaller in the Q sample ( $<0.6 \%)$ than in the S sample $(0.8 \%)$ and reported Li-rich layered materials ( $\sim 1.2 \%$ change after $1^{\text {st }}$ cycle). ${ }^{38,39}$ The difference can be a result of the different degree of cation disordering in the layered structure of the samples. Considering that the $c / a$ ratio of the layered phase after cycles is related to the degree of cation disordering (or mixing), ${ }^{38,39}$ increased cation disordering in the layered phase in the pristine Q sample can lead to negligible change in the $c / a$ ratio during cycles due to high layered structure stability. ${ }^{40}$ This result indicates that the degree of the cation disordering barely increases in the Ni incorporated $\mathrm{Li}_{2} \mathrm{MnO}_{3}$-like layered phase in the $\mathrm{Q}$ sample during 2 cycles compared to the $\mathrm{S}$ sample because $\mathrm{Ni}$ in $\mathrm{Li}$ layers in the initially partially cation-disordered $\mathrm{Li}_{2} \mathrm{MnO}_{3}$-like layered phase help in stabilizing the layered structure even with almost full extraction of $\mathrm{Li}$ by reducing strong electrostatic repulsion between TM layers. (Supplementary Table. 4) Robust layered structure induced by the increase in cation disordering ( $\mathrm{Ni}$ incorporation in Li layer) in pristine layered structure and resilient spinel structure induced by defective structural features such as $\mathrm{Ni} / \mathrm{Mn}$ disordering with oxygen and Ni vacancies can help the Q sample to enable the Mn-rich spinellayered composite material to achieve high reversible energy density (Fig. 4b) and high power density simultaneously (Fig. 4f).

\section{Discussion}

In this study, the limited solubility of $\mathrm{Ni}$ and $\mathrm{O}$ atoms in the Mn-rich electrode materials achieved at high temperature can alter the composition of spinel and $\mathrm{Li}_{2} \mathrm{MnO}_{3}$ layered ideal 
domains and induces the formation of a composite, which is composed of a defective spinel and a partially cation-disordered layered phase containing Ni. The resulting materials can deliver both high reversible capacity, $340 \mathrm{mAh} / \mathrm{g}$ and high rate capability up to $10 \mathrm{C}$ rate (3A/g) simultaneously. The limited solubility of atoms such as Ni and oxygen in the LNMO spinel phase at high temperature $\left(>700{ }^{\circ} \mathrm{C}\right)$ leads to the formation of the rocksalt $\mathrm{Li}_{\mathrm{x}} \mathrm{Ni}_{\mathrm{y}} \mathrm{O}$ phase ${ }^{7-9}$ that can react with the $\mathrm{Li}_{2} \mathrm{MnO}_{3}$ layered phase. This reaction caused by limited solubility of the atoms can form defective LNMO-like spinel phase and the Ni incorporated $\mathrm{Li}_{2} \mathrm{MnO}_{3}$-like layered phase. Firstly, the defective LNMO-like spinel phase can substantially improve rate capability in the Mn-rich materials by reducing the structural change even after insertion of large amount of Li below 3V, where usually Li ions can be inserted into empty 16c sites and typically inducing severe structural changes caused by a phase transition to the tetragonal spinel phase. ${ }^{21}$ The $\mathrm{Ni}$ vacancies and oxygen vacancies along with the increased $\mathrm{Ni} / \mathrm{Mn}$ disordering in the defective spinel phase can enlarge local structures around empty $16 \mathrm{c}$ sites that can easily accommodate structural changes resulting in the stabilization of the cubic spinel phase. Secondly, the $\mathrm{Ni}$ incorporated $\mathrm{Li}_{2} \mathrm{MnO}_{3}$-like layered phase can achieve high reversible capacity in the Mn-rich materials by enhancing oxygen redox reaction in the layered phase partly because $\mathrm{Ni}$ can stabilize the oxygen redox reaction. ${ }^{29,30}$ Also, the increase in the cation disordering ( $\mathrm{Ni}$ in Li layers) in the layered phase can mitigate the intrinsic layered structure instability caused by electrostatic repulsive force between layers and thereby can allow full extraction of $\mathrm{Li}^{40}$ As a result, controlled composite structure can achieve both high energy density and high power capability even with a wide voltage range in the Mn-rich electrode materials.

In Mn-rich electrode materials, previous approaches for improving electrochemical performance have been focused on increasing the energy density rather than power density, by 
using double $\mathrm{Mn}$ redox reactions in the disordered rocksalt structure, ${ }^{2,3}$ which can have intrinsically poor Li kinetics, or typical composite of the spinel-layered phase like the S sample, ${ }^{4,6}$ which shows severe structural changes induced by a phase transition in the spinel phase. Compared to these previous approaches, controlled atomic solubility in a composite structure can be an effective and efficient way to achieve both high energy density and high power density simultaneously in Mn-rich electrode materials.

In summary, we demonstrate that controlled atomic solubilities in a composite of Mn-rich electrode materials can achieve both high energy density and high power density simultaneously. The controlled composite structure is composed of (i) a defective spinel phase, that can accommodate large amount of Li while mitigating severe structural distortion resulting in facile kinetics and (ii) a $\mathrm{Ni}$ incorporated layered phase that can enhance reversible electrochemical activity due to the positive $\mathrm{Ni}$ role on oxygen redox activity in layered materials. Our findings can unlock the potential of Mn-rich electrode materials and thereby can provide the possibility of their use in large-scale energy storage systems in which cost and availability of raw materials are critical limitations. This strategy of achieving both high energy density and high power by controlling atoms solubility in the composite structure of Mn-rich materials will open new avenues for designing electrode materials that have abundant raw materials and achieve high electrochemical performance for large-scale Li-ion batteries.

\section{Methods}

Preparation of materials. This spinel-layered composite was synthesized by solid-state reaction in four steps. (1) Appropriate ratios of $\mathrm{Li}_{2} \mathrm{CO}_{3}, \mathrm{MnO}_{2}$, and $\mathrm{NiCO}_{3}$ were ball-milled in acetone for $12 \mathrm{~h}$. These samples had the molar ratio of $\mathrm{Li}: \mathrm{Ni}: \mathrm{Mn}=1.0: 0.16: 0.84$. (2) A mix of precursors was pelletized, then calcined at $900{ }^{\circ} \mathrm{C}$ for $6 \mathrm{~h}$ in air. (3) The calcined pellets were 
grounded and pulverized by planetary ball-milling (PBM, Fritsch Pulverisette planetary ballmill) for 3 h. (A synthesized sample that had not been subjected to PBM can show poor oxygen redox activity due to insufficient interaction between $\mathrm{Li}_{2} \mathrm{MnO}_{3}$ and $\mathrm{LiNi}_{0.5} \mathrm{Mn}_{0.5} \mathrm{O}_{2}$ at high temperature even after a quenching process.) The pulverized powder was re-pelletized. (4) The pellets were reannealed for $5 \mathrm{~h}$ in air at $800{ }^{\circ} \mathrm{C}$, then either quenched to RT in air to yield the Q sample, or cooled naturally to RT to yield the S sample.

Material Characterizations. The Neutron Diffraction measurements were performed at the Australian Centre for Neutron Scattering. The wavelength was $1.5340 \AA$ and the scan range was $10^{\circ}$ to $150^{\circ}$ in increments of 0.05 . All data were collected at RT. The synchrotron X-ray diffraction measurements were performed on beamline 9B-HRPD at Pohang Accelerator Laboratory, Pohang, Korea. The incident X-rays were vertically collimated by a mirror, and then monochromated to the wavelength of $1.4970 \AA$ by a double-crystal Si (111) monochromator. The datasets were collected in the range of $10^{\circ} \leq 2 \theta \leq 130^{\circ}$ with a step size of $0.02^{\circ}$ ( $2 \theta$ range). The lattice parameter of each sample and the quantity of impurities were determined using Full Proof software. High-resolution transmission electron microscopy (TEM) analysis was conducted on a JOEL JEM-2200FS microscope fitted with a LaB6 filament at an acceleration voltage of 200kV. Rietveld refinement was done using FULLPROF software.

Nuclear Magnetic Resonance (NMR) Measurements. ${ }^{7} \mathrm{Li}$ NMR measurements were performed at RT on a Bruker Avance-200 spectrometer $\left(\mathrm{B}_{0}=4.7 \mathrm{~T}\right.$, Larmor frequency $v_{0}=$ 77.78 MHz). Magic Angle Spinning (MAS) spectra were obtained by using a Bruker MAS 
probe with a cylindrical 2.5-mm (o.d.) zirconia rotor. Spinning speed was varied between 25, 30, and $34 \mathrm{kHz}$ in order to determine the position of the isotropic resonances. ${ }^{7} \mathrm{Li}$ MAS NMR spectra were acquired using an echo $(\pi / 2-\tau-\pi-\tau)$ pulse sequence with a $\pi / 2$ pulse of $2.3 \mu \mathrm{s}$. Recycle time was typically 0.5s. The isotropic shifts, reported in parts per million (ppm), are relative to an external liquid $1 \mathrm{M}$ solution of $\mathrm{LiCl}$ set at $0 \mathrm{ppm}$.

X-ray Absorption Spectroscopy (XAS) Measurements. Soft XAS spectra of O K-edge were recorded at beamline 10A - XAS KIST of Pohang Light Source II in total electron yield mode and fluorescence yield mode under high-vacuum (base pressure 3 x $10^{-10}$ Torr). The spectral energy resolution was $0.1 \mathrm{eV}$ and the monochromator absorption features were normalized by dividing the detected signals $I_{1}$ by the photoemission current $I_{0}$ of a gold mesh placed in the incident beam. All absorption spectra were collected at RT.

Electrochemical measurements._For electrochemical tests, composite electrodes were made by mixing active material (80 wt\%), super-P carbon (Timcal, $15 \mathrm{wt} \%$ ), and binder (Polyvinylidene fluoride, $5 \mathrm{wt} \%$ ). A slurry mixture was tape-cast on Al foil by the doctor-blade method. The loading density of the electrode was $2-4 \mathrm{mg} / \mathrm{cm}^{2}$. We assembled the cells in an Argon-filled glove box and tested them on a Maccor 2200 operating in galvanostatic mode using Lithium metal as an anode, non-aqueous electrolyte (1 $\mathrm{M} \mathrm{LiPF}_{6}$ in ethylene carbonate (EC): Diethyl carbonate (DEC) (1:1 by volume, PANAX ETEC Co. Ltd., battery grade), and Celgard 2400 as a separator in a 2032-coin cell. All cells were tested at RT.

\section{References}


1 Chu, S., Cui, Y. \& Liu, N. The path towards sustainable energy. Nature materials 16, 16 (2017).

2 Lee, J. et al. Reversible Mn 2+/Mn 4+ double redox in lithium-excess cathode materials. Nature 556, 185 (2018).

3 Freire, M. et al. A new active Li-Mn-O compound for high energy density Li-ion batteries. Nature materials 15, 173 (2016).

4 Bhaskar, A. et al. Synthesis and Characterization of High-Energy, High-Power SpinelLayered Composite Cathode Materials for Lithium-Ion Batteries. Advanced Energy Materials 5, 1401156 (2015).

5 Cabana, J., Kang, S.-H., Johnson, C. S., Thackeray, M. M. \& Grey, C. P. Structural and electrochemical characterization of composite layered-spinel electrodes containing $\mathrm{Ni}$ and Mn for Li-ion batteries. Journal of The Electrochemical Society 156, A730-A736 (2009).

6 Lee, E.-S., Huq, A., Chang, H.-Y. \& Manthiram, A. High-voltage, high-energy layeredspinel composite cathodes with superior cycle life for lithium-ion batteries. Chemistry of Materials 24, 600-612 (2012).

7 Song, J. et al. Role of Oxygen Vacancies on the Performance of Li [Ni0. 5-x Mn1. 5+ x] O4 ( $\mathrm{x}=0,0.05$, and 0.08) Spinel Cathodes for Lithium-Ion Batteries. Chemistry of Materials 24, 3101-3109 (2012).

8 McCalla, E., Rowe, A., Shunmugasundaram, R. \& Dahn, J. Structural study of the LiMn-Ni oxide Pseudoternary system of interest for positive electrodes of Li-ion batteries. Chemistry of Materials 25, 989-999 (2013).

9 Choi, H. W. et al. Temperature-dependent oxygen behavior of LixNi0. 5Mn1. $5 \mathrm{O} 4$ cathode material for lithium battery. APL Materials 4, 116111 (2016).

10 Kim, J.-H. et al. Integrated nano-domains of disordered and ordered spinel phases in LiNi0. 5Mn1. $5 \mathrm{O} 4$ for Li-Ion batteries. Chemistry of Materials 26, 4377-4386 (2014).

11 Grey, C. P. \& Dupré, N. NMR studies of cathode materials for lithium-ion rechargeable batteries. Chemical Reviews 104, 4493-4512 (2004).

12 Grey, C. P. \& Lee, Y. J. Lithium MAS NMR studies of cathode materials for lithiumion batteries. Solid State Sciences 5, 883-894 (2003).

13 Cabana, J. et al. Composition-structure relationships in the Li-ion battery electrode material LiNi0. 5Mn1. 5O4. Chemistry of Materials 24, 2952-2964 (2012).

14 Lee, J., Dupre, N., Avdeev, M. \& Kang, B. Understanding the cation ordering transition 
in high-voltage spinel LiNi 0.5 Mn 1.5 O 4 by doping Li instead of Ni. Scientific reports 7, 6728 (2017).

15 Buzlukov, A. et al. Li-Rich Mn/Ni Layered Oxide as Electrode Material for Lithium Batteries: A 7Li MAS NMR Study Revealing Segregation into (Nanoscale) Domains with Highly Different Electrochemical Behaviors. The Journal of Physical Chemistry C 120, 19049-19063 (2016).

16 Ben, L. et al. Unusual Spinel-to-Layered Transformation in LiMn2O4 Cathode Explained by Electrochemical and Thermal Stability Investigation. ACS applied materials \& interfaces 9, 35463-35475 (2017).

17 Pearce, P. E. et al. Evidence for anionic redox activity in a tridimensional-ordered Lirich positive electrode $\beta$-Li 2 IrO 3. Nature materials 16, 580 (2017).

18 Zheng, J. et al. Enhanced Li+ ion transport in LiNi $0.5 \mathrm{Mn} 1.5 \mathrm{O} 4$ through control of site disorder. Physical Chemistry Chemical Physics 14, 13515-13521 (2012).

19 Zheng, J. et al. Mitigating voltage fade in cathode materials by improving the atomic level uniformity of elemental distribution. Nano letters 14, 2628-2635 (2014).

20 Yu, X. et al. Understanding the Rate Capability of High-Energy-Density Li-Rich Layered Li1. 2Ni0. 15Co0. 1Mn0. $55 \mathrm{O} 2$ Cathode Materials. Advanced Energy Materials 4, 1300950 (2014).

21 Lee, E.-S., Nam, K.-W., Hu, E. \& Manthiram, A. Influence of cation ordering and lattice distortion on the charge-discharge behavior of LiMn1. 5Ni0. $5 \mathrm{O} 4$ spinel between 5.0 and 2.0 V. Chemistry of Materials 24, 3610-3620 (2012).

22 Park, S. et al. Comparative study of different crystallographic structure of LiNi0. 5Mn1. $504-\delta$ cathodes with wide operation voltage (2.0-5.0 V). Electrochimica Acta 52, 7226-7230 (2007).

23 Höweling, A. et al. Influence of synthesis, dopants and cycling conditions on the cycling stability of doped LiNi0. 5Mn1. $5 \mathrm{O} 4$ spinels. Journal of The Electrochemical Society 164, A6349-A6358 (2017).

24 Hy, S. et al. Performance and design considerations for lithium excess layered oxide positive electrode materials for lithium ion batteries. Energy \& Environmental Science 9, 1931-1954 (2016).

25 Oishi, M. et al. Direct observation of reversible charge compensation by oxygen ion in Li-rich manganese layered oxide positive electrode material, Li $1.16 \mathrm{Ni} 0.15$ Co 0.19 
Mn 0.50 O 2. Journal of Power Sources 276, 89-94 (2015).

$26 \mathrm{Xu}$, J. et al. Elucidating anionic oxygen activity in lithium-rich layered oxides. Nature communications 9, 947 (2018).

27 Yoon, W.-S. et al. Investigation of the Charge Compensation Mechanism on the Electrochemically Li-Ion Deintercalated Li1-x Co1/3Ni1/3Mn1/3O2 Electrode System by Combination of Soft and Hard X-ray Absorption Spectroscopy. Journal of the American Chemical Society 127, 17479-17487 (2005).

28 Luo, K. et al. Charge-compensation in 3d-transition-metal-oxide intercalation cathodes through the generation of localized electron holes on oxygen. Nature chemistry $\mathbf{8}, 684$ 691 (2016).

29 Hy, S. et al. Understanding the Role of Ni in Stabilizing the Lithium-Rich HighCapacity Cathode Material Li [Ni x Li (1d electron holes on oxygen. stem byChemistry of Materials 26, 6919-6927 (2014).

30 Hoang, K. Doping Li-rich cathode material Li $2 \mathrm{MnO}$ 3: Interplay between lattice site preference, electronic structure, and delithiation mechanism. Physical Review Materials 1, 075404 (2017).

31 Nayak, P. K. et al. Improved capacity and stability of integrated Li and Mn rich layeredspinel Li 1.17 Ni 0.25 Mn 1.08 O 3 cathodes for Li-ion batteries. Journal of Materials Chemistry A 3, 14598-14608 (2015).

32 Kosilov, V., Potapenko, A. \& Kirillov, S. Effect of overdischarge (overlithiation) on electrochemical properties of LiMn 2 O 4 samples of different origin. Journal of Solid State Electrochemistry 21, 3269-3279 (2017).

33 Nayak, P. K. et al. Electrochemical performance of a layered-spinel integrated Li [Ni1/3Mn2/3] O2 as a high capacity cathode material for Li-ion batteries. Chemistry of Materials 27, 2600-2611 (2015).

34 Fell, C. R. et al. Correlation between oxygen vacancy, microstrain, and cation distribution in lithium-excess layered oxides during the first electrochemical cycle. Chemistry of Materials 25, 1621-1629 (2013).

35 Piszora, P., Nowicki, W. \& Darul, J. High-pressure metaelastic properties of Li x Mn 3- x O 4 (x=0.87, 0.94, 1.00). Journal of Materials Chemistry 18, 2447-2452 (2008).

36 Peterson, V. Lattice parameter measurement using Le Bail versus structural (Rietveld) refinement: A caution for complex, low symmetry systems. Powder Diffraction 20, 1417 (2005). 
37 Le Bail, A., Duroy, H. \& Fourquet, J. Ab-initio structure determination of LiSbWO6 by X-ray powder diffraction. Materials Research Bulletin 23, 447-452 (1988).

38 Shen, C.-H. et al. Facile synthesis of the Li-rich layered oxide Li1. 23Ni0. 09Co0. 12Mn0. $56 \mathrm{O} 2$ with superior lithium storage performance and new insights into structural transformation of the layered oxide material during charge-discharge cycle: In situ XRD characterization. ACS applied materials \& interfaces 6, 5516-5524 (2014).

39 Liu, H. et al. Operando Lithium Dynamics in the Li-Rich Layered Oxide Cathode Material via Neutron Diffraction. Advanced Energy Materials 6, 1502143 (2016).

40 Myeong, S. et al. Understanding voltage decay in lithium-excess layered cathode materials through oxygen-centred structural arrangement. Nature communications $\mathbf{9}$, 3285 (2018).

\section{Acknowledgements}

This research was supported by the Basic Science Research Program through the National Research Foundation of Korea (NRF) funded by the Ministry of Science, ICT \& Future Planning (NRF-2015M2A2A6A01044985 and NRF-2017M3A7B8065394). This research was supported by the Brain Korea 21 PLUS Project for Center for Creative Industrial Materials (F14SN02D1707) and a POSTECH Basic Science Research Institute Grant.

\section{Author contributions}

B. K and J. L brought the idea and design of the experiments, and in drafting the article for important intellectual content. Q.Z. and L.G. performed and analyzed the TEM imaging experiments; N.D. contributed to analysis and interpretation of NMR data and M.A. contributed to analysis of Neutron diffraction data. W.-S. Y and M. J performed the soft X-ray absorption spectroscopy and analyses. All of the authors discussed the results and reviewed the manuscript.

\section{Additional information}


Supplementary information is available online. Reprints and permissions information is available online at www.nature.com/reprints. Correspondence and requests for materials should be addressed to B. K.

\section{Competing interests}

The authors declare no competing financial interests.
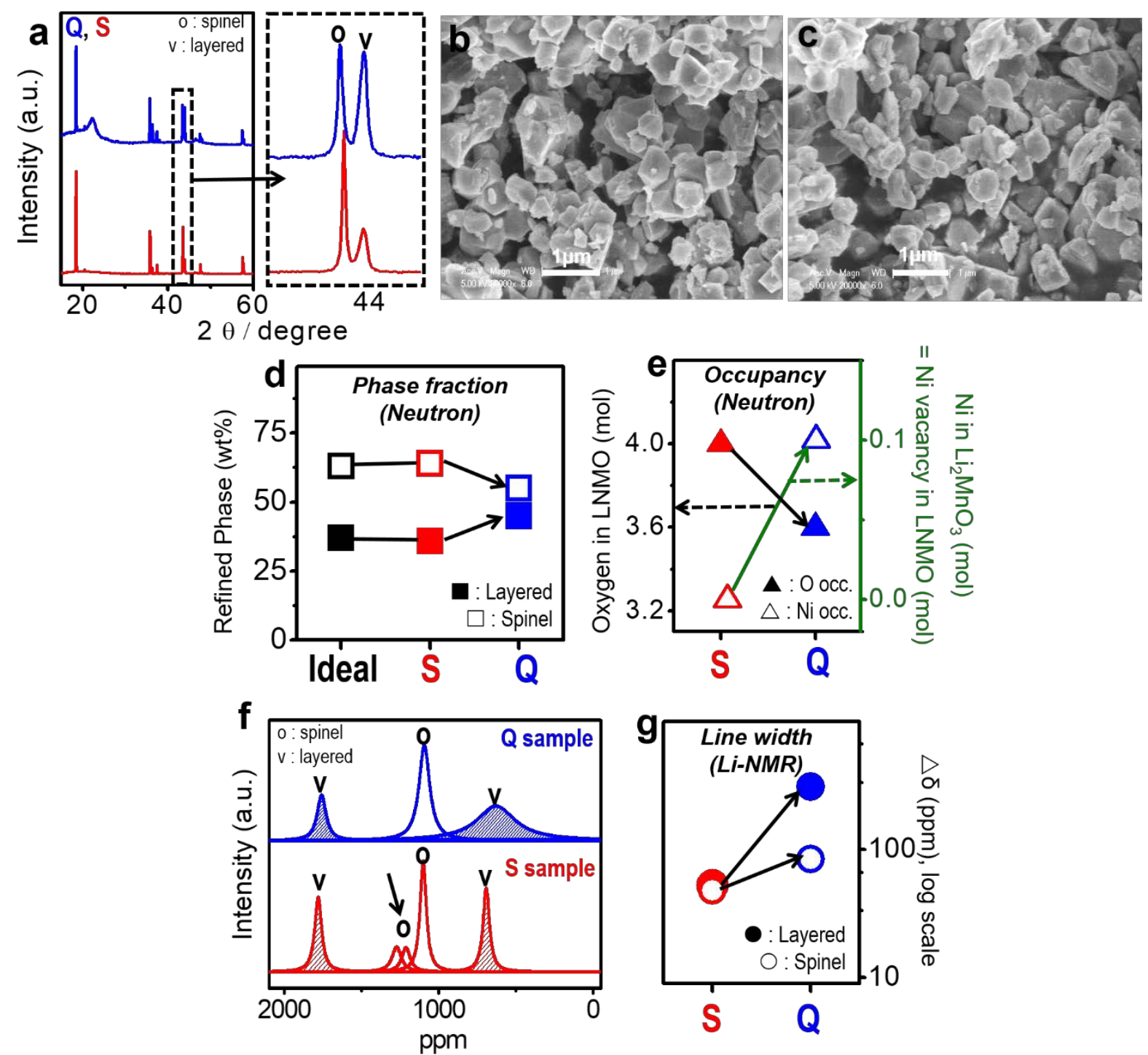

Figure. 1. Structural characterizations of the samples. a, Synchrotron XRD diffraction patterns of the two samples. (v: layered; $\mathrm{Li}_{2} \mathrm{MnO}_{3}$, o : spinel; LNMO) SEM images of $\mathbf{b}, \mathrm{Q}$ sample and c, S sample. d, Phase fraction of LNMO spinel (Fd-3m) phase and $\mathrm{Li}_{2} \mathrm{MnO}_{3}(\mathrm{C} / 2 \mathrm{~m})$ 
layered phase in the samples, as determined by Rietveld refinement of Neutron powder diffraction e, Site occupancies of the oxygen atoms in LNMO spinel (Fd-3m) phase and the Ni atoms in the $\mathrm{Li}_{2} \mathrm{MnO}_{3}(\mathrm{C} / 2 \mathrm{~m})$ phase in the samples, that is the same as Ni vacancies in LNMO (Fd-3m) spinel phase as determined by Rietveld refinement of Neutron powder diffraction. (Supplementary Fig. 1 and Supplementary Table 2) f, Deconvoluted lines obtained from ${ }^{7} \mathrm{Li}$ MAS NMR at spinning speeds of $34 \mathrm{kHz}$ for $\mathrm{Li}$ local environments in $\mathrm{Li}_{2} \mathrm{MnO}_{3}$, layered and LNMO spinel in the Q and S samples. Only isotropic lines are displayed for clarity purpose. g, Apparent linewidth $\Delta \delta$ (ppm) based on deconvoluted lines obtained from ${ }^{7} \mathrm{Li}$ MAS NMR at spinning speeds of $34 \mathrm{kHz}$ (Supplementary Table 3). 


\section{a}

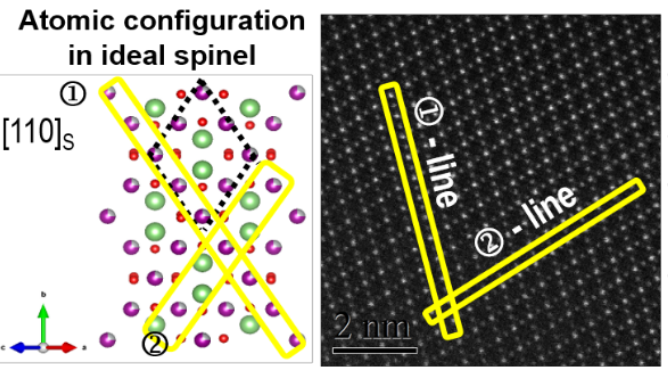

C Atomic configuration

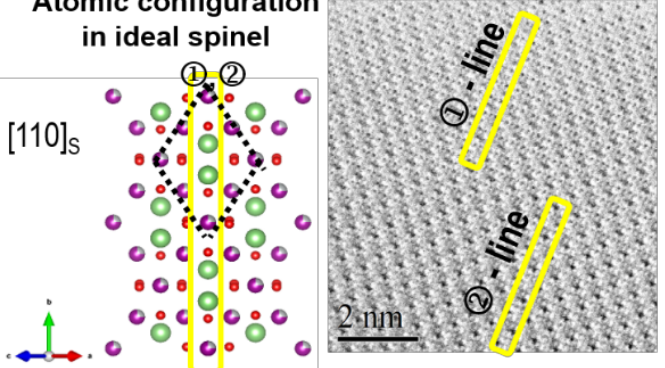

e Atomic configuration

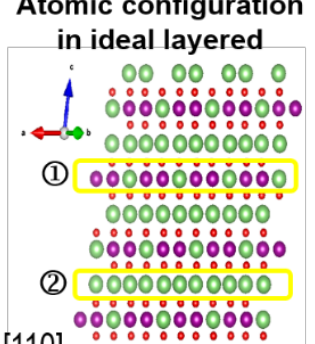
[110] L 000000

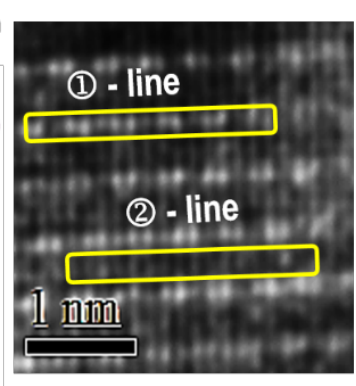

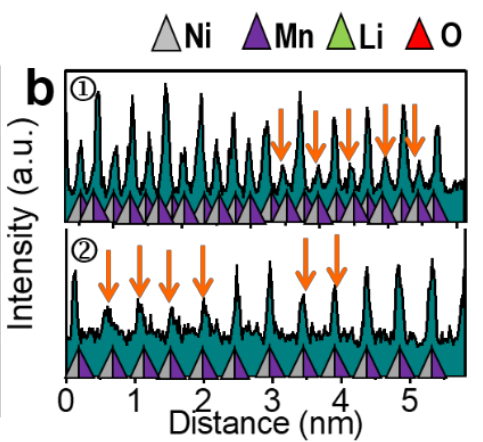
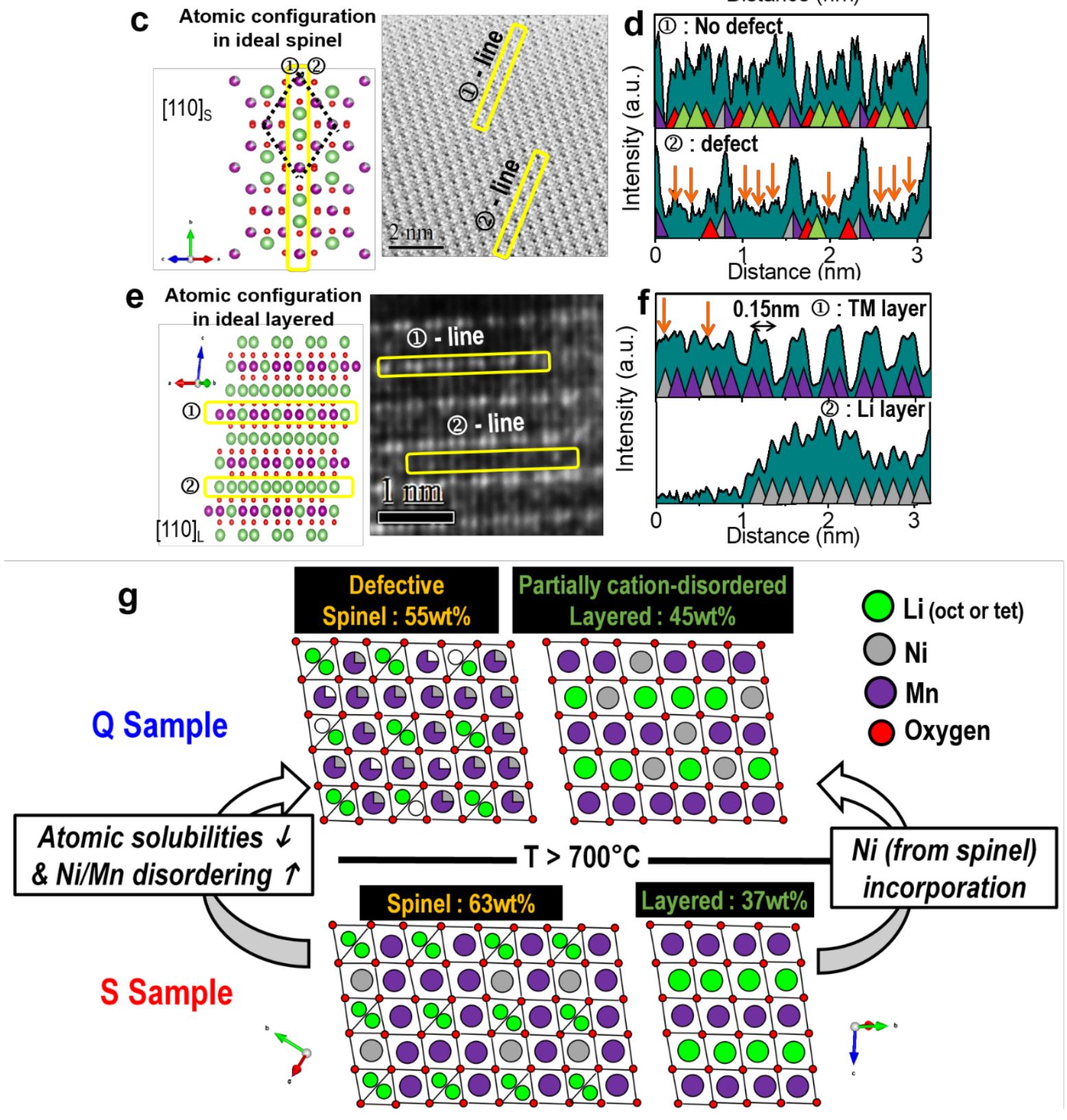

Figure. 2. Local structures of the two phases in the composite of the $\mathbf{Q}$ sample. Local structure of the defective $\mathrm{LiNi}_{0.5} \mathrm{Mn}_{1.5} \mathrm{O}_{4}$ spinel-like phase in $\mathrm{Q}$ sample a, Atomic configuration in a unit cell of LNMO $\left(\mathrm{LiNi}_{0.5} \mathrm{Mn}_{1.5} \mathrm{O}_{4}\right)$ spinel structure and HAADF image projected along 
the [110] zone axis b, HAADF image intensity profile with the two different spinel regions along the [110] direction (yellow box in a). c, Atomic configuration in a unit cell of LNMO $\left(\mathrm{LiNi}_{0.5} \mathrm{Mn}_{1.5} \mathrm{O}_{4}\right)$ spinel structure and ABF image projected along the [110] zone axis d, The ABF image intensity profile with different two spinel regions along the [110] direction (yellow box in c). Local structure of the $\mathrm{Ni}$ incorporated $\mathrm{Li}_{2} \mathrm{MnO}_{3}$-like layered phase in the $\mathrm{Q}$ sample e, Atomic configuration in a unit cell of ideal $\mathrm{Li}_{2} \mathrm{MnO}_{3}$ layered structure and HAADF image projected along the [110] zone axis. f, HAADF image intensity profile in Li layer (2), yellow box in e) and TM layer (1), yellow box in e) (Grey: Ni, Purple: Mn, Red: Oxygen, Green: Li) g, Schematic diagrams of possible local atomic configurations in the spinel-layered composite in the samples, Q sample: defective spinel-partially disordered (cation mixing) layered composite and S sample: typical spinel-layered composite. 

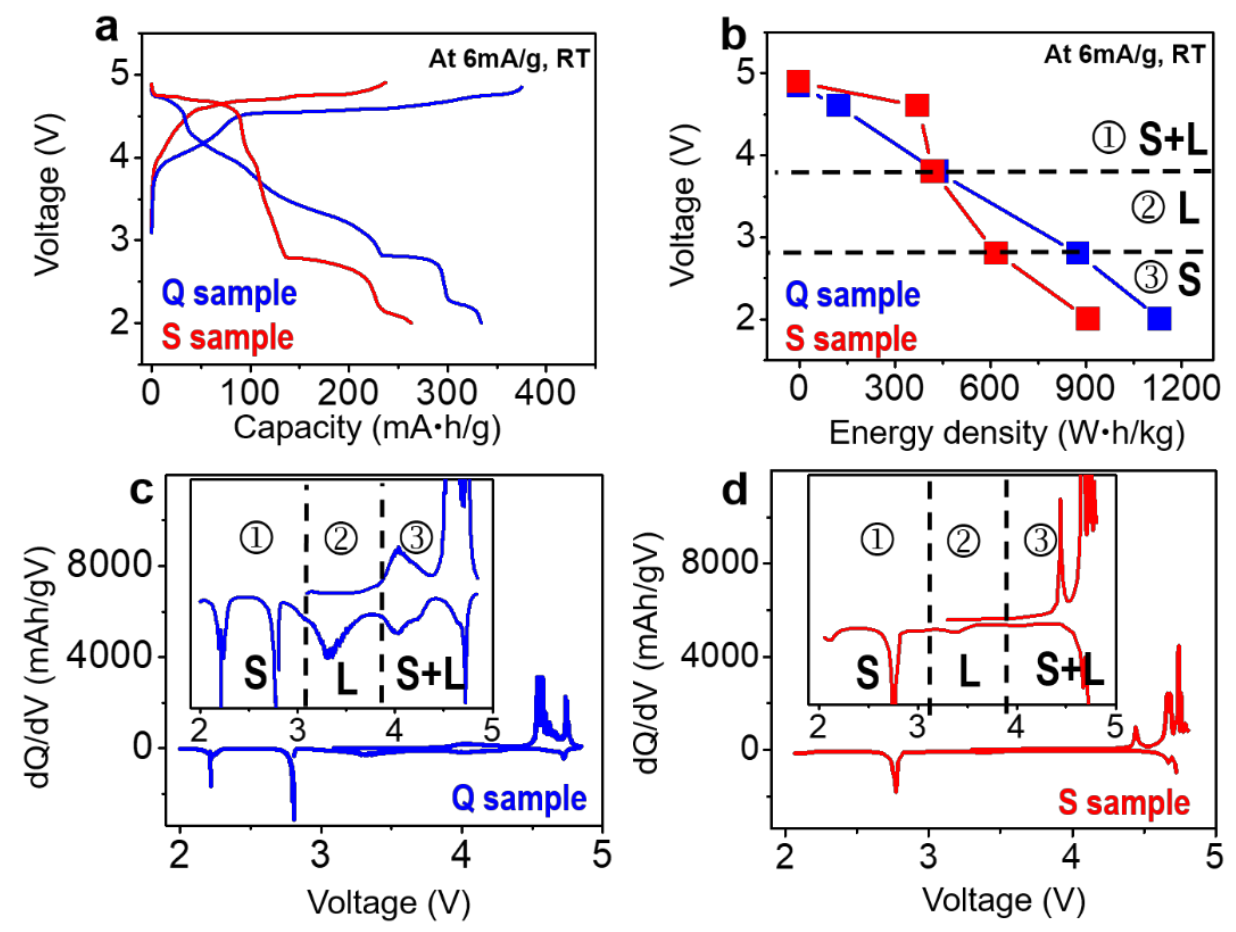

Figure. 3. Electrochemical properties of the two samples. a, Voltage curves of the Q sample and the S sample at 2.0 to $4.9 \mathrm{~V}$ and $6.0 \mathrm{~mA} / \mathrm{g}$ at RT (from Galvanostatic test). b, Voltage vs. energy density plot of the Q sample and S sample at 2.0 to $4.9 \mathrm{~V}$ and $6.0 \mathrm{~mA} / \mathrm{g}$ at RT (from Galvanostatic test). Differential capacity plots, $\mathrm{d} Q / \mathrm{d} V$ obtained from voltage profiles in Fig. 3a for c, Q sample and d, S sample. (S: Spinel phase, L: Layered phase) 

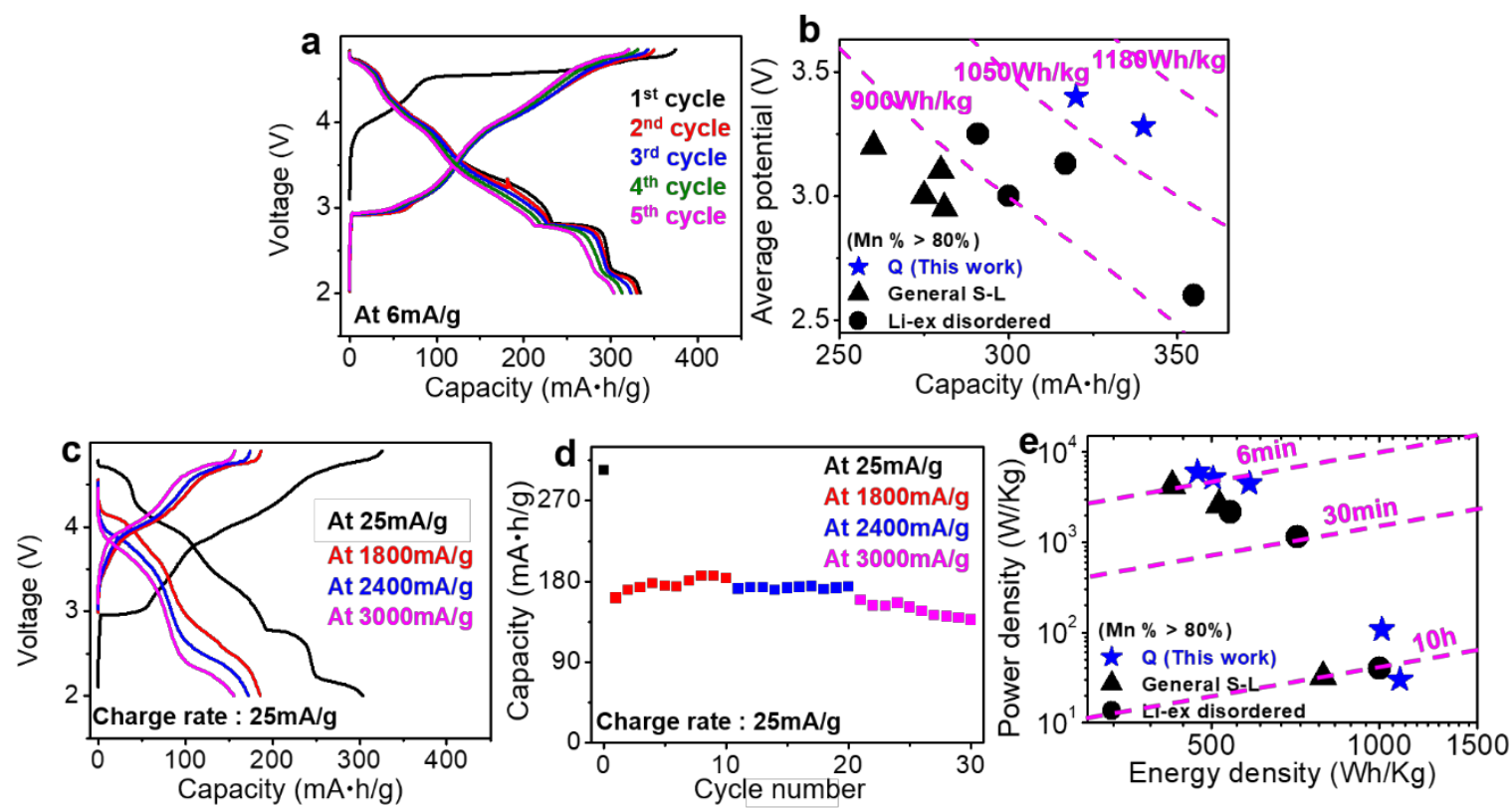

Figure. 4. Electrochemical performance of the $\mathbf{Q}$ sample. a, Voltage curves for 5 cycles in the Q sample at C/50 (6 mA/g) at RT b, Ragone plot of energy density among Mn-rich electrode materials (Mn involved above 80\% of total transition metals in composition) and the Q sample. Rate capability test of the Q sample c, Voltage profiles at different rates. d, Capacity retentions at different rates. e, Capacity retentions at 2C charge - 2C discharge rate (600 mA/g). f, Ragone plot of power density among Mn-rich electrode materials. All electrochemical tests were performed at RT at an operating voltage window $2.0 \mathrm{~V}$ to $4.9 \mathrm{~V} ; 1 \mathrm{C}=300 \mathrm{~mA} / \mathrm{g}$. 

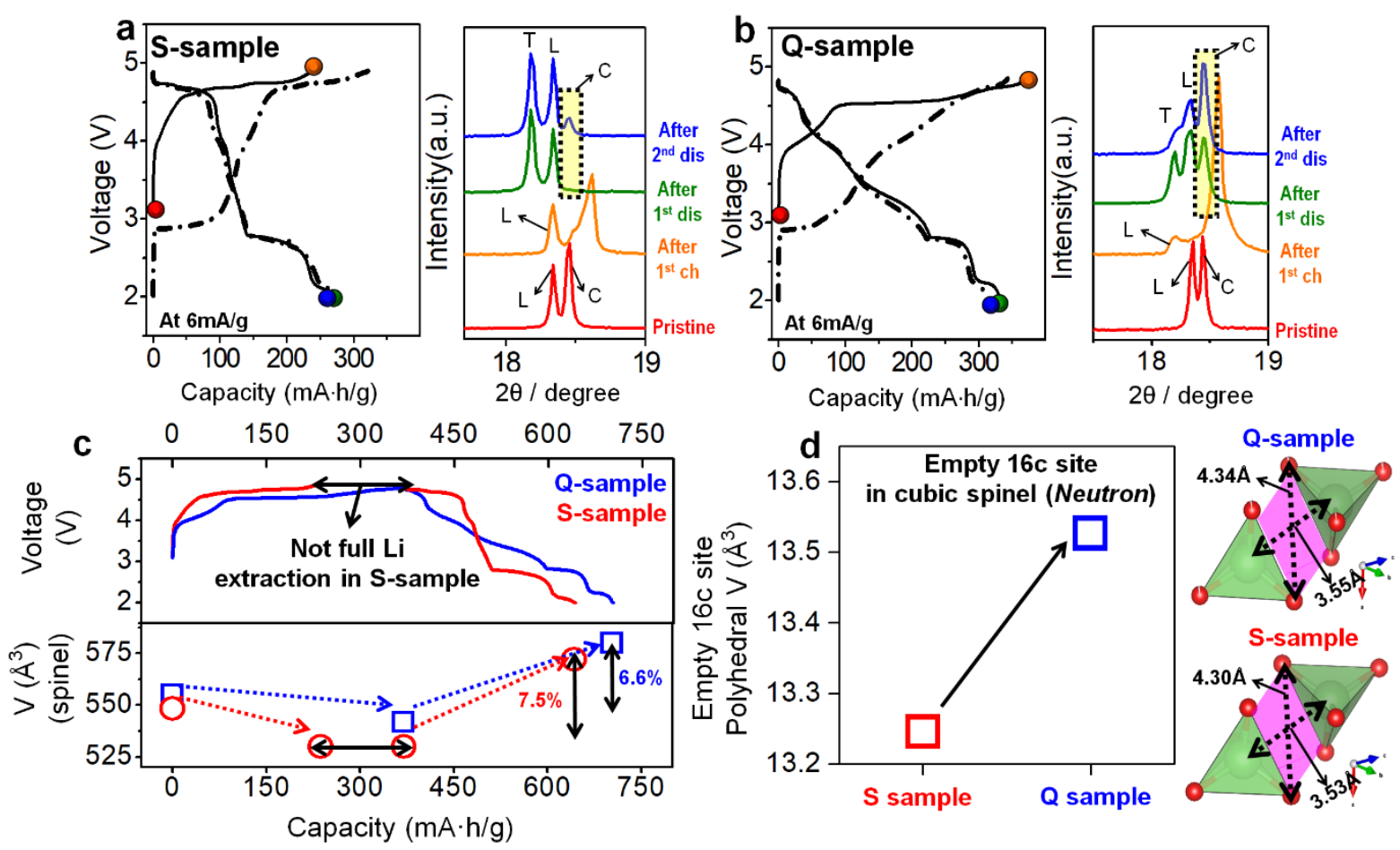

Figure. 5. Structural changes in the two samples during two cycles. Voltage profiles at $\mathrm{C} / 50$ rate and Ex-situ Synchrotron XRD data of the electrodes during 2 cycles of $\mathbf{a}$, the S sample and b, the Q sample. c, Voltage profile at C/50 rate and volume changes for the spinel phase in the S sample and the Q sample from Le Bail refinements of Ex-situ Synchrotron XRD data of the electrodes d, Polyhedral volume (pink shade) of empty 16c site in pristine samples determined by Rietveld refinement of Neutron powder diffraction $\left(\mathrm{L}=\mathrm{Li}_{2} \mathrm{MnO}_{3}\right.$ layered, $\mathrm{C}=$ cubic $\mathrm{LNMO}$ spinel, $\mathrm{T}=$ tetragonal LNMO spinel) 